\title{
Wang Koch-adjusted axial length in SRK/T formula for ocular biometry in high myopia: a prospective study
}

\author{
Fouad Chraibi ®D, Hassan Moutei, Ahmed Bennis, Meriem Abdellaoui, Idriss Andaloussi Benatiya \\ University Hospital Hassan II and University Sidi Mohamed Ben Abdellah, Fez, Morocco
}

\begin{abstract}
BACKGROUND: The aim of this study was to prospectively assess refractive results of cataract surgery in highly myopic eyes using the SRKT formula and Wang Koch-adjusted axial length.

MATERIAL AND METHODS: Prospectively, we recruited consecutive candidates for cataract surgery having an axial length equal to $27 \mathrm{~mm}$ and longer. We performed biometry by using Wang and Koch-adjusted axial length applied to the SRKT formula. The main outcome measures were: mean of refractive error, mean of absolute refractive error, and percentage of eyes that achieved a refractive error of $\pm 0.5 \mathrm{D}$ and $\pm 1 \mathrm{D}$.

RESULTS: Fifteen eyes of nine patients were involved in the study. The mean refractive error was $-0.01 \mathrm{D} \pm 0.4 \mathrm{D}$, and the mean absolute refractive error was $+0.35 \mathrm{D} \pm 0.20 \mathrm{D}$. Refractive errors of $\pm 0.5 \mathrm{D}$ and $\pm 1 \mathrm{D}$ were achieved, respectively, in $86.6 \%$ and in $100 \%$ of eyes.

CONCLUSIONS: Wang Koch's axial length adjustment applied to the SRKT formula is a reliable alternative in high myopic cataract patients.
\end{abstract}

KEY WORDS: cataract; high myopia; Wang Koch adjustment; SRK/T formula; refractive error; absolute refractive error

Ophthalmol J 2020; Vol. 5, 12-16

\section{INTRODUCTION}

Phacoemulsification is a functional rehabilitation surgery but also, and increasingly, a refractive surgery. Eye biometry is an important step in the process. There are still some challenging cases of eye biometry such as in cases of keratoconus, after refractive surgery, and in cases of extreme axial length.

In the present paper, we focus on eye biometry in high myopic cataract surgery candidates. Conventional formulas used to perform biometry tend to underestimate the power of implant in cases of high myopia yielding postoperative hypermetropia [1].
In high myopia, part of the problem is that the measurement of the axial length (AL) is inaccurate, particularly in the case of associated myopic staphyloma. Optical biometry can partially compensate for this issue but does not completely alleviate it because of certain intrinsic deficiencies of the used formulas.

The new generation of formulas, including Barrett Universal II and Hill-RBF, enable the best results but necessitate optical biometry - a technology not available in all centres, especially in developing countries. 
For this reason, we analyse prospectively Wang Koch's axial length optimisation method for the SRK/T formula in high myopic cataract surgery candidates.

\section{MATERIAL AND METHODS}

This study was conducted following the declarations of Helsinki and after obtaining the patient's informed consent.

This was a prospective study that spanned from November 2018 to August 2019; all patients with high myopia (AL $\geq$ to $27 \mathrm{~mm}$ ) and who are candidates to cataract surgery by phacoemulsification had had ultrasonic or optical (Lenstar Ls 900; Haag-Streit AG, Koeniz, Switzerland) eye biometry depending on the transparency of the media.

Following the recommendations of Wang et al., we used the constant $\mathrm{a}=118.4$ as advocated by the ULIB (User Group for Laser Interference Biometry) optimised for the formula SRK/T and adapted to the implant that we used.

Keratometry was measured either with an automatic refractometer or during the acquisition of optical ocular biometry. Patients with keratometric astigmatism of over $1.5 \mathrm{D}$ were excluded from the study.

In patients with high myopia, the recommended post-operative target refraction is $-0.75 \mathrm{D}$ to $-3 \mathrm{D}$, or even up to $-5 \mathrm{D}$ in cases of high myopia with macular lesion [2]. In our series and after discussion with our patients, the refractive target was $-2 \mathrm{D}$, except for one patient who preferred emmetropia as postoperative target refraction.

The initial calculation of the intraocular lens power (IOLm; $\mathrm{m}$ for measured) was performed based on the SRK/T formula using the measured axial length. In the second step, the second calculation of adjusted IOL power (IOLa; a for adjusted) was based on a computed axial length according to the method of Wang Koch for the SRK/T formula:

$$
\begin{gathered}
\text { Adjusted axial length }=0.8453 \times \text { measured axial } \\
\text { length }+4.0773 \mathrm{~mm} .
\end{gathered}
$$

For implants with intermediate steps of $0.5 \mathrm{D}$, which are not provided by the manufacturer in the range of powers from $-10 \mathrm{D}$ to $+10 \mathrm{D}$, we increased the value of the power of the used IOL (IOLu; $\mathrm{u}$ for used) by $+0.5 \mathrm{D}$ (example: if the power of IOLa $=-2.5 \mathrm{D}$, which is not available, the power of IOLu will be -2D).

All patients are operated by coaxial phacoemulsification by the same surgeon (FC), with a $2.4 \mathrm{~mm}$ upper temporal incision and implantation of a hydrophobic acrylic implant (Eyecryl Plus, Biotech) for all patients. No early intraoperative or postoperative complications occurred. On the other hand, we excluded cases that required a stitch at the end of the surgery for its probable effect on the induced astigmatism and final spherical equivalent.

Postoperative refraction was assessed two months after surgery.

The main measured outcomes are the average of the refractive error, the average of the absolute refractive error, the percentage of refractive errors between $-0.5 \mathrm{D}$ and $+0.5 \mathrm{D}( \pm 0.5 \mathrm{D})$, and the percentage of refractive errors between $-1 \mathrm{D}$ and $+1 \mathrm{D}$ $( \pm 1 \mathrm{D})$.

The biometric refractive error is equal to the postoperative refraction (spherical equivalent) minus the targeted refraction. The absolute biometric refractive error corresponds to the difference between the postoperative refraction and the target refraction in absolute value. The calculation of the mean of the absolute biometric refractive errors makes it possible to assess the precision of the measurements of the formula used [3].

\section{RESULTS}

The total number of eyes was 15 eyes of nine patients whose ages ranged from 42 to 56 years with an average of 52 years; the sex ratio was 0.8 ( 4 men and 5 women).

The measured axial length was on average $30.44 \mathrm{~mm} \pm 2.35 \mathrm{~mm}$ (from $27.18 \mathrm{~mm}$ to $34.37 \mathrm{~mm}$ ) (Tab. 1), and the adjusted axial length according to Wang Koch's formula was $29.81 \mathrm{~mm} \pm 1.99 \mathrm{~mm}$ (from $27.05 \mathrm{~mm}$ to $33.13 \mathrm{~mm}$ ). The average of the power of IOLm was $5.66 \mathrm{D} \pm 4.98 \mathrm{D}$ (from $-1.75 \mathrm{D}$ to $+12.5 \mathrm{D})$, and the average power of the IOLa was $7.23 \mathrm{D} \pm 4.28 \mathrm{D}$ (from $1 \mathrm{D}$ to $13.5 \mathrm{D}$ ).

The mean of the refractive error was $-0.01 \mathrm{D} \pm 0.4 \mathrm{D}$, ranging from $-0.5 \mathrm{D}$ to $+0.75 \mathrm{D}$, with an overall trend towards relative emmetropia.

The mean of the absolute refractive error was $+0.35 \mathrm{D} \pm 0.20 \mathrm{D}$, ranging from 0 to $+0.75 \mathrm{D}$, confirming the high precision of the adjusted formula 


\begin{tabular}{|c|c|}
\hline Parameter & Value \\
\hline Number of eyes & 15 \\
\hline \multicolumn{2}{|l|}{ Age (years) } \\
\hline Average \pm SD & $52 \pm 4.7$ \\
\hline Extremes & $42-56$ \\
\hline \multicolumn{2}{|l|}{ Sex } \\
\hline Female & 5 \\
\hline Male & 4 \\
\hline Sex ratio & 0.8 \\
\hline \multicolumn{2}{|l|}{ Axial length [mm] } \\
\hline Average $\pm S D$ & $30.44 \pm 2.35$ \\
\hline Extremes & $27.18-34.37$ \\
\hline \multicolumn{2}{|l|}{ Power LIOm [D] } \\
\hline Average \pm SD & $5.66 \pm 4.98$ \\
\hline Extremes & -1.75 to +12.5 \\
\hline
\end{tabular}

$S D$ - standard deviation; $L 10 \mathrm{~m}$ - measured power of the artificial lens

(Tab. 2). The percentage of the refractive error within $\pm 0.5 \mathrm{D}$ was $86.6 \%$ and within $\pm 1 \mathrm{D}$ in $100 \%$ of cases.

\section{DISCUSSION}

In 2015, L. Wang and D. Koch proposed a method for optimising ocular biometry formulas in high myopia by modifying the axial length according to the following original formula [4]:

\section{Optimised axial length $=0.8981 \times A L$} (in $\mathrm{mm}$ ) $+2.5637 \mathrm{~mm}$.

However myopic refractive results were of significant proportion, and these same authors proposed in 2018 a new regression for the adjustment of the axial length in SRK/T, as follows:

\section{Axial length optimised $=0.8453 \times$ measured axial length $+4.0773 \mathrm{~mm}[5]$.}

In our study, the average of biometric refractive error was almost zero (a trend toward relative emmetropia), and the average of the absolute biometric refractive error was $+0.33 \mathrm{D} \pm 0.21 \mathrm{D}$. The percentage of postoperative refractions were within $\pm 0.5 \mathrm{D}$ and $\pm 1 \mathrm{D}$ in $86.6 \%$ and $100 \%$ of cases, respectively. Yokoi et al. [6], using the SRK/T formula without adjustment of the axial length in

\begin{tabular}{|l|c|c|c|c|c|c|c|}
\hline Table 2. The different parameters before and after adjusting the axial length \\
\hline Eye & LAm & LAa & LIOm & LIOa & Liu & MRE & MARE \\
\hline 1 & 33.52 & 32.41 & -1.75 & 1 & 1 & -0.25 & 0.25 \\
\hline 2 & 33.41 & 32.31 & -1.5 & 1 & 1 & 0.50 & 0.50 \\
\hline 3 & 27.34 & 27.18 & 11.5 & 12 & 12 & -0.25 & 0.25 \\
\hline 4 & 27.18 & 27.05 & 11 & 11.5 & 11.5 & 0.00 & 0.00 \\
\hline 5 & 29.89 & 29.34 & 6.5 & 8 & 8 & 0.50 & 0.50 \\
\hline 6 & 29.57 & 29.07 & 7 & $8.5^{*}$ & 9 & -0.50 & 0.50 \\
\hline 7 & 34.37 & 33.13 & -1 & $1.5^{*}$ & 2 & 0.25 & 0.25 \\
\hline 8 & 34.22 & 33.00 & -1.5 & 1 & 1 & 0.75 & 0.75 \\
\hline 9 & 30.54 & 29.89 & 4.5 & 6 & 6 & 0.25 & 0.25 \\
\hline 10 & 30.87 & 30.17 & 6 & $7.5^{*}$ & 8 & -0.25 & 0.25 \\
\hline 11 & 28.67 & 28.31 & 10.5 & 11.5 & 11.5 & -0.25 & 0.25 \\
\hline 12 & 28.49 & 28.15 & 10 & 11 & 11 & -0.25 & 0.25 \\
\hline 13 & 28.14 & 27.86 & 12.5 & 13.5 & 13.5 & -0.25 & 0.25 \\
\hline 14 & 31.12 & 30.38 & 3 & 5 & 5 & 0.75 & 0.75 \\
\hline 15 & 29.34 & 28.87 & 8.5 & 9.5 & 10 & -0.25 & 0.25 \\
\hline Avg & 30.44 & 29.81 & 5.68 & 7.23 & 7.36 & -0.01 & 0.35 \\
\hline SD & 02.35 & 1.99 & 4.98 & 4.28 & 4.27 & 0.40 & 0.20 \\
\hline
\end{tabular}

LAm — axial length measured in $\mathrm{mm}$; LAa — axial length adjusted in $\mathrm{mm}$; LIOm — power of the implant measured in $\mathrm{D}$; LIOa — power of the implant adjusted in $\mathrm{D}$; Liu — power of the implant used in D; MRE — mean refractive error; MARE — the mean of the absolute refractive error; Avg — average; SD — standard deviation; " not available 
high myopic patients (AL $\geq 26.5 \mathrm{~mm}$ ) in 84 eyes, found an average postoperative biometric refractive error of $+0.45 \mathrm{D} \pm 0.79 \mathrm{D}$ (hypermetropic shift) with an average of the absolute refractive error of $+0.72 \mathrm{D} \pm 0.47 \mathrm{D}$; and a postoperative refraction of $\pm 1 \mathrm{D}$ in only $70 \%$ of cases.

On the other hand, our refractive results meet the standards set by the study of the Swedish $\mathrm{Na}$ tional Register of cataract surgery establishing postoperative reactive values of $\pm 0.5 \mathrm{D}$ in at least $71.0 \%$ of the eyes and of $\pm 1.0 \mathrm{D}$ in $93.0 \%$ of the cases as the optimal results [7].

Among all the currently available formulas in high myopia, the benchmark is represented by the Barrett Universal II formula, which according to the literature provides the lowest average postoperative refractive error $(-0.09 \mathrm{D} \pm 0.42 \mathrm{D}$ to $+0.05 \mathrm{D} \pm 0.46 \mathrm{D})$ and the best percentage of a postoperative refraction of $\pm 0.5 \mathrm{D}(77 \%)$ [8-11]. We consider that the results obtained in our series using adjustment of the axial length according to the Wang Koch method are close to the performance of the Barrett Universal II formula, which is rather modern and sophisticated but which requires technology not yet available for routine practice in developing countries.

Third- and fourth-generation formulas (SRKT, Hoffer Q, Holiday I and II) tend to underestimate the power of the implant with consequent hyperopia. This outcome is more noticeable in eyes with an axial length $\geq$ at $31 \mathrm{~mm} \mathrm{[6]} \mathrm{and} \mathrm{in} \mathrm{cases} \mathrm{of} \mathrm{nega-}$ tive IOL power [12].

Aboulafia et al. [13]. state that in highly myopic patients with an axial length of more than $26 \mathrm{~mm}$, a threshold value of IOL power of $6 \mathrm{D}$ allows segregation of high myopic patients into two groups at different risk of postoperative hyperopic refractive error. Those having less than $6 \mathrm{D}$ as IOL power will have a higher risk of postoperative refractive error. Indeed, in their assessment of the SRK/T formula, the average refractive error was close to $0 \mathrm{D}(-0.05 \mathrm{D} \pm 0.35 \mathrm{D})$ in the group of IOL powers of $6 \mathrm{D}$ and more, while in the group of IOL powers less than $6 \mathrm{D}$ the average refractive error was $+0.82 \mathrm{D} \pm 0.53 \mathrm{D}$. After adjusting the axial length according to the Wang Koch method applied to SRK/T, the average refractive error was $-0.31 \mathrm{D} \pm 0.36 \mathrm{D}$ for group $6 \mathrm{D}$ and more and $+0.02 \mathrm{D} \pm 0.49 \mathrm{D}$ for the group of less $6 \mathrm{D}$.

Our study is certainly prospective but nevertheless has certain limits. First, the small size of our series due to numerous self-imposed restrictions during the process of patient recruitment for the study. Second, the lack of homogeneity of measurements given the use of optical biometry and ultrasound depending on the transparency of the media in the same series. And finally, the unavailability by the manufacturer of some intermediate IOL power values with steps of $+0.5 \mathrm{D}$ in the range $-10 \mathrm{D}$ to $+10 \mathrm{D}$.

\section{CONCLUSION}

Wang Koch's axial length adjustment for high myopia cataract candidates is a reliable alternative to the more sophisticated formulas such as Barrett Universal II. Special attention is advised regarding eyes with an axial length of $\geq 27 \mathrm{~mm}$, particularly those with an $A L \geq 31 D$, as well as in cases of IOL power $<6 \mathrm{D}$, particularly those of negative IOL power values.

\section{Conflict of interests}

The authors declare that they have no conflict of interests in relation to this article.

\section{REFERENCES}

1. Zuberbuhler B, Seyedian M, Tuft S. Phacoemulsification in eyes with extreme axial myopia. J Cataract Refract Surg. 2009; 35(2): 335-340, doi: 10.1016/j.jcrs.2008.10.044, indexed in Pubmed: 19185252.

2. Hayashi K, Hayashi H. Optimum target refraction for highly and moderately myopic patients after monofocal intraocular lens implantation. J Cataract Refract Surg. 2007; 33(2): 240-246, doi: 10.1016/j. jcrs.2006.10.045, indexed in Pubmed: 17276264.

3. Hoffer KJ, Aramberri J, Haigis W, et al. Protocols for studies of intraocular lens formula accuracy. Am J Ophthalmol. 2015; 160(3): 403-405.e1, doi: 10.1016/j.ajo.2015.05.029, indexed in Pubmed: 26117311.

4. Wang Li, Shirayama M, Ma XJ, et al. Optimizing intraocular lens power calculations in eyes with axial lengths above $25.0 \mathrm{~mm}$. J Cataract Refract Surg. 2011; 37(11): 2018-2027, doi: 10.1016/j.jcrs.2011.05.042, indexed in Pubmed: 22018365.

5. Wang Li, Koch DD. Modified axial length adjustment formulas in long eyes. J Cataract Refract Surg. 2018; 44(11): 1396-1397, doi: 10.1016/j.jcrs.2018.07.049, indexed in Pubmed: 30274847.

6. Yokoi T, Moriyama M, Hayashi $\mathrm{K}$, et al. Evaluation of refractive error after cataract surgery in highly myopic eyes. Int Ophthalmol. 2013; 33(4): 343-348, doi: 10.1007/s10792-012-9690-6, indexed in Pubmed: 23315193.

7. Behndig A, Montan P, Stenevi U, et al. Aiming for emmetropia after cataract surgery: Swedish National Cataract Register study. J Cataract Refract Surg. 2012; 38(7): 1181-1186, doi: 10.1016/j. jcrs.2012.02.035, indexed in Pubmed: 22727287.

8. Wang 0 , Jiang Wu, Lin T, et al. Accuracy of intraocular lens power calculation formulas in long eyes: a systematic review and metaanalysis. Clin Exp Ophthalmol. 2018; 46(7): 738-749, doi: 10.1111/ ceo.13184, indexed in Pubmed: 29498180.

9. Melles RB, Holladay JT, Chang WJ. Accuracy of Intraocular Lens Calculation Formulas. Ophthalmology. 2018; 125(2): 169-178, doi: 10.1016/j.ophtha.2017.08.027, indexed in Pubmed: 28951074.

10. Liu J, Wang Li, Chai F, et al. Comparison of intraocular lens power calculation formulas in Chinese eyes with axial myopia. J Cataract Refract Surg. 2019; 45(6): 725-731, doi: 10.1016/j.jcrs.2019.01.018, indexed in Pubmed: 31146930. 
11. Zhou D, Sun Z, Deng G. Accuracy of the refractive prediction determined by intraocular lens power calculation formulas in high myopia. Indian J Ophthalmol. 2019; 67(4): 484-489, doi: 10.4103/ ijo.IJO 937 18, indexed in Pubmed: 30900579.

12. Zaldivar R, Shultz MC, Davidorf JM, et al. Intraocular lens power calculations in patients with extreme myopia. J Cataract Refract Surg.
2000; 26(5): 668-674, doi: 10.1016/s0886-3350(00)00367-9, indexed in Pubmed: 10831895.

13. Abulafia A, Barrett GD, Rotenberg M, et al. Intraocular lens power calculation for eyes with an axial length greater than $26.0 \mathrm{~mm}$ : comparison of formulas and methods. J Cataract Refract Surg. 2015; 41(3): 548-556, doi: 10.1016/j.jcrs.2014.06.033, indexed in Pubmed: 25708208. 